\title{
Modeling Reorientation Dynamics of Electrically Assisted Light-Induced Gliding of Nematic Liquid-Crystal Easy Axis
}

\author{
S. V. Pasechnik, ${ }^{1,2}$ A. V. Dubtsov, ${ }^{1}$ D. V. Shmeliova, ${ }^{1}$ D. A. Semerenko, ${ }^{1}$ \\ V. G. Chigrinov, ${ }^{2}$ M. A. Sinenko, ${ }^{3}$ and Alexei D. Kiselev ${ }^{2,4}$ \\ ${ }^{1}$ Moscow State University of Instrument Engineering and Computer Science, Stromynka 20, Moscow 107846, Russia \\ ${ }^{2}$ Hong Kong University of Science and Technology, Clear Water Bay, Kowloon, Hong Kong \\ ${ }^{3}$ Chernigov State Technological University, Shevchenko Street 95, Chernigov 14027, Ukraine \\ ${ }^{4}$ Institute of Physics of National Academy of Sciences of Ukraine, Prospekt Nauki 46, Kiev 03028, Ukraine
}

Correspondence should be addressed to Alexei D. Kiselev; kiselev@iop.kiev.ua

Received 18 May 2013; Revised 12 August 2013; Accepted 14 August 2013

Academic Editor: Kim Chow

Copyright (C) 2013 S. V. Pasechnik et al. This is an open access article distributed under the Creative Commons Attribution License, which permits unrestricted use, distribution, and reproduction in any medium, provided the original work is properly cited.

\begin{abstract}
The phenomenological torque balance model previously introduced to describe the electrically assisted light-induced gliding is generalized to study the reorientation dynamics of the nematic liquid crystal easy axis at photoaligned azo-dye films under the combined action of in-plane electric field and reorienting UV light linearly polarized at varying polarization azimuth, $\varphi_{p}$. We systematically examine the general properties of the torque balance model by performing analysising the bifurcations of equilibria at different values of the polarization azimuth and apply for the model to interpret the experimental results. These involve observation of the pronounced purely photoinduced reorientation at $\varphi_{p} \neq 0$, as opposed to the case where the light polarization vector is parallel to the initial easy axis $\left(\varphi_{p}=0\right)$, and the reorientation is almost entirely suppressed. In the regions between electrodes with nonzero electric field, the effects described by the model are that (a) the dynamics of reorientation slows down with $\varphi_{p}$ and (b) the sense of easy axis rotation is independent of the sign of $\varphi_{p}$.
\end{abstract}

\section{Introduction}

It is well known that the orientational structure of a nematic liquid crystal (NLC) placed in contact with an anisotropic substrate is essentially determined by the properties of the interfacial region, where various kinds of surface-induced ordering may exist (see, e.g., [1-3] for reviews). At the macroscopic level, the surface-induced orientation of NLC molecules in the interfacial layer manifest, itself as the well-known phenomenon anchoring which can be roughly described as the tendency of the nematic director $\mathbf{n}_{s}$ to align along the direction of preferential anchoring orientation at the surface. The direction of surface-induced alignment is specified by a unit vector $\mathbf{n}_{e}$ and is referred to as the easy axis.

Anchoring is governed by the so-called anchoring energy, $W_{\text {anch }}$, which is the orientationally dependent (anisotropic) part of the surface tension. In particular, easy axes can be found by minimizing the anchoring potential and, thus, crucially depend on the shape of $W_{\text {anch. When the anchoring }}$ energy changes, the easy axes may vary in both direction and number. Such variations of the anchoring conditions result in reorientation of the NLC director known as the anchoring (surface) transition.

Since the anchoring potential is sensitive to the thermodynamic parameters, the anchoring transitions, similar to the phase transitions, can be driven by temperature, chemical potential, and strain. Of particular interest are the transitions governed by the parameters that characterize the method used to treat the surface for fabrication of aligning films. There are photo-induced orientational surface transitions [46] that are related to the photoalignment (PA) technique, which is employed in the manufacturing process of liquid crystal displays for fabricating high quality aligning substrates and uses linearly polarized ultraviolet (LPUV) light to induce anisotropy of the angular distribution of molecules in azo-dye containing photosensitive films [7]. 
In the PA method, the easy axis is determined by the polarization azimuth of the pumping LPUV light, whereas the azimuthal and polar anchoring strengths may depend on a number of governing parameters such as the wavelength and the irradiation dose $[8,9]$.

So, in an NLC cell with the initially irradiated layer, subsequent illumination with reorienting light which polarization differs from the one used to prepare the layer can trigger the light-induced reorientation of the easy axis which is governed by the photoinduced reordering of azo-dye molecules [9] and may be of considerable interest for applications such as LC rewritable devices [10]. Note that slow reorientation of the easy axis-the so-called electric-field-induced easy axis gliding-on the photosensitive layers prepared using the PA technique was originally observed on poly-(vinyl)-alcohol (PVA) coatings with embedded azo-dye molecules [11]. For azo-dye films, similar results were reported in [12].

Clearly, the effects of the polarization azimuth, $\varphi_{p}$, that characterizes orientation of the polarization vector of reorienting LPUV light, $\mathbf{E}_{\mathrm{UV}}$, play a key role in the process of lightinduced easy axis reorientation and will be of our primary concern. More specifically, we consider how the polarization azimuth affects the reorientational dynamics of the electrically assisted light-induced azimuthal gliding of the easy axis that takes place on photoaligned azo-dye layers when irradiation of nematic LC (NLC) cells with LPUV light is combined with the application of ac in-plane electric field [13].

It was observed that, at certain combinations of the parameters such as the amplitude of electric field, $E$, the light intensity, $I_{\mathrm{UV}}$, the exposure time, $t_{\mathrm{exp}}$, and the dose of the initial UV irradiation, $D_{p}$, the switching off relaxation considerably slows down up to few months [13]. The switching on dynamics of the gliding for both the linearly polarized and the nonpolarized reorienting light was studied in [14]. In particular, the results of the papers $[13,14]$ demonstrate that the combined effect may be used as a tool to tune technical parameters of LC memory devices. So, as compared to the case of purely light-induced reorientation of the easy axis governed by the effect of photoinduced ordering in azo-dye layers, the dynamics of the electrically assisted light-induced gliding can be additionally influenced by the electric field, $E$.

In previous studies $[13,14]$, the reorienting light was linearly polarized along the initial easy axis. The theoretical considerations of [9] are based on the diffusion model describing the light-induced reordering in azo-dye films as rotational Brownian motion of azo-dye molecules governed by the lightintensity dependent mean-field potential. These thus deal with the case of the purely light-induced reorientation. For this limiting case where the effects of the in-plane electric field are negligible, the results of [9] suggest the generalization of the torque balance model [12-14] that will be of our main concern.

Our overall goal is to study how the polarization azimuth influences the surface-mediated reorientation processes that occur in NLC cells under the combined action of LPUV light and in-plane electric field. By contrast to our recent brief report [15], in this study, most attention has been concentrated on the modeling of the electrically assisted light-induced gliding and on the problems related to interpretation of the experimental data.

The layout of the paper is as follows.

In Section 2, we formulate our phenomenological torque balance model of electrically assisted photoinduced easy axis reorientation that takes into account the effects due to the polarization azimuth. Then, for the dynamical system representing the model, the regime of photosaturation is studied by analyzing the bifurcations of equilibria at different values of the polarization azimuth.

In Section 3, after short description of the experimental procedure used to measure the azimuthal easy axis angle as a function of irradiation time, the theoretical curves computed from the model are compared with the experimental data measured at various values of the polarization azimuth.

Finally, in Section 4 we discuss the results and make some concluding remarks.

\section{Torque Balance Model}

In this section, we carry out a theoretical investigation into the effects of the reorienting light polarization by using a generalized version of the phenomenological model formulated in [12-14] to describe the effect of electrically assisted light-induced azimuthal gliding. According to this model, the anchoring characteristics of the photoaligned layer such as the easy axis, $\mathbf{n}_{e}$, are determined by orientational order of LC molecules adsorbed by the azo-dye film.

Initially, after PA treatment made before the cell is filled with LC, ordering of the adsorbed molecules is dictated by the orientational order parameter of the azo-dye layer induced by the initial irradiation with linearly polarized UV light, $\mathbf{E}_{0}$. The initial direction of preferential orientation at the surface $\mathbf{n}_{0}$ is typically normal to the principal axis of the photoinduced anisotropy defined by the polarization vector $\mathbf{E}_{0}$.

When the cell filled with LC is subsequently irradiated by the linearly polarized reorienting light $\mathbf{E}_{\mathrm{UV}}$ with the polarization azimuth which differs from the one used at the preparation stage for PA treatment of the azo-dye layer, absorbed LC molecules undergo light-induced transitions. These transitions occur due to reorientation of azo-dye molecules interacting with the adsorbed LC layer. Thus, this is the dynamics of photoinduced reordering of azo-dye molecules that underlies the effect of the reorienting light on the anchoring properties of the photoaligned layer [9].

The easy axis can also be influenced by applying an inplane electric field $\mathbf{E}$ in the direction perpendicular to $\mathbf{n}_{0}$. This can be explained in terms of adsorption-desorption processes taking place in the near-surface layer.

Initially, in the absence of electric field, the surface director $\mathbf{n}_{s}$ characterizing average orientation of LC molecules in the near-surface layer is directed along the easy axis $\mathbf{n}_{0}$. So, the adsorption-desorption processes do not influence the undisturbed angular distributions of LC molecules in the absorbed and near-surface layers which are initially identical.

An electric field $\mathbf{E}$ produces a twist deformation on the distance $\xi$ defined as the electric coherence length, $\xi=$ $(1 / E) \sqrt{K_{t} /\left(\varepsilon_{0} \Delta \varepsilon\right)}$, where $\Delta \varepsilon$ is the electric permittivity anisotropy and $K_{t}$ is the effective Frank elastic constant for 
the twist deformation. At $\Delta \varepsilon>0$, under this electricfield-induced deformation, the surface director, $\mathbf{n}_{s}$, inclines towards the electric field $\mathbf{E} \perp \mathbf{n}_{0}$ (on the reference substrate). The absorption-desorption processes involving exchange of molecules between the differently aligned (absorbed and near-surface) layers will result in reorientation of absorbed molecules. Note that, owing to low probability of adsorptiondesorption events, noticeable changes may require very long periods of time. A detailed microscopic justification of the absorption-desorption mechanism responsible for the easy axis gliding in the presence of light and electric field can be found in [13].

In the phenomenological model $[12,13]$, orientation of the easy axis $\mathbf{n}_{e}$ characterized by the azimuthal angle $\varphi_{e}$ is defined by the balance of the three torques: the torque arising from the reorientation of azo-dye molecules, the torque transmitted from the bulk by the near-surface layer, and the viscous torque proportional to the specific viscosity of gliding $\gamma_{e}$. For the surface director $\mathbf{n}_{s}$ with the azimuthal angle $\varphi_{s}$, the analogues balance involves the torque arising due to deviation of the surface director from the easy axes (it is proportional to the surface anchoring energy strength $W_{s}$ ), the torque transmitted from the bulk, and the viscous torque proportional to the surface viscosity $\gamma_{s}$.

The resulting system of balance torque equations for the easy axis and surface director azimuthal angles, $\varphi_{e}$ and $\varphi_{s}$, reads

$$
\begin{aligned}
& \gamma_{e} \frac{\partial \varphi_{e}}{\partial t}=K_{E}\left(\frac{\pi}{2}-\varphi_{s}\right)-\frac{W_{e}}{2} \sin 2\left(\varphi_{e}-\varphi_{m}\right), \\
& \gamma_{s} \frac{\partial \varphi_{s}}{\partial t}=K_{E}\left(\frac{\pi}{2}-\varphi_{s}\right)-\frac{W_{s}}{2} \sin 2\left(\varphi_{e}-\varphi_{s}\right),
\end{aligned}
$$

where $K_{E}$ is the electric field induced torque coefficient inversely proportional to the electric coherence length $\xi$ and $W_{e}$ is the effective anchoring parameter which defines the strength of coupling between the easy axis $\mathbf{n}_{e}$ and the initial state of surface orientation described by the vector $\mathbf{n}_{0}$.

An important additional parameter is the phase shift $\varphi_{m}$ that enters the second term on the right-hand side of (1a) and depends on the polarization azimuth of the reorienting light. In the field-free regime with $E=0$ and $K_{E}=0$, (1a) assumes the simplified form

$$
\frac{\partial \varphi_{e}}{\partial t}=-\frac{W_{e}}{2 \gamma_{e}} \sin 2\left(\varphi_{e}-\varphi_{m}\right),
$$

giving the formula for the easy axis angle

$$
\tan \left(\varphi_{e}(t)-\varphi_{m}\right)=\tan \left(\varphi_{e}(0)-\varphi_{m}\right) \exp \left[-\frac{t}{t_{e}}\right],
$$

where $t_{e}=\gamma_{e} / W_{e}$ is the characteristic time of purely photoinduced easy axis reorientation that represents the solution of (2). This formula describes the phase shift as the azimuthal angle characterizing the photosteady orientation of the easy $\operatorname{axis} \varphi_{e}^{(\mathrm{st})}=\varphi_{m}\left(\varphi_{e}^{(\mathrm{st})}=\varphi_{m}+\pi / 2\right)$ at $W_{e}>0\left(W_{e}<0\right)$. Interestingly, in our recent paper [9], it was shown that, under certain assumptions, (2) can be derived from the diffusion model of photoinduced reordering in azo-dye films. In this case, it turned out that the phase shift $\varphi_{m}$ equals the angle between the polarization vector of the reorienting light, $\mathbf{E}_{\mathrm{UV}}$, and the axis directed along the normal to $\mathbf{n}_{0}$.

Note that the model previously treated in [12-14] corresponds to the special cases with either $\varphi_{m}=0$ or $\varphi_{m}=\pi / 2$. In subsequent sections, the effects due to variations of the phase shift will be of our primary interest.

2.1. Bifurcation Analysis of Dynamical System. Before making comparison between the model and experiment, we will dwell briefly on the general properties of the dynamical system (1a) and (1b) which can be conveniently recast into the following dimensionless form:

$$
\begin{gathered}
\frac{\partial \varphi_{e}}{\partial \tau}=\psi_{s}-w_{e} \sin 2\left(\varphi_{e}-\varphi_{m}\right) \equiv \Phi\left(\varphi_{e}, \psi_{s}\right), \\
\frac{\partial \psi_{s}}{\partial \tau}=\gamma\left\{-\psi_{s}+w_{s} \sin 2\left(\varphi_{e}+\psi_{s}\right)\right\} \equiv \gamma \Psi\left(\varphi_{e}, \psi_{s}\right), \\
\tau=\frac{t}{\tau_{e}}, \quad \psi_{s} \equiv \frac{\pi}{2}-\varphi_{s},
\end{gathered}
$$

where $\tau_{e}=\gamma_{e} / K_{E}$ is the characteristic time of easy axis reorientation; $\gamma=\gamma_{e} / \gamma_{s}$ is the viscosity ratio; $w_{e}=W_{e} /\left(2 K_{E}\right)$ and $w_{s}=W_{s} /\left(2 K_{E}\right)$ are the dimensionless anchoring parameters. The two symmetry relations

$$
\begin{gathered}
\varphi_{m} \longrightarrow \varphi_{m}+\frac{\pi}{2} \Longleftrightarrow w_{e} \longrightarrow-w_{e}, \\
\varphi_{m} \longrightarrow-\varphi_{m} \Longleftrightarrow\left\{\varphi_{e}, \psi_{s}\right\} \longrightarrow\left\{-\varphi_{e},-\psi_{s}\right\}
\end{gathered}
$$

describe how the system (4a) and (4b) transforms when the phase shift is translated by $\pi / 2$ (the polarization vector is rotated by $\pi / 2$ around the $z$-axis which is normal to the layer), and the phase shift changes its sign (the polarization vector is reflected with respect to the $y$-axis).

The equilibrium (photosteady) states characterized by the azimuthal angles, $\varphi_{e}^{(\mathrm{st})}=\pi / 2-\psi_{e}^{(\mathrm{st})}$ and $\psi_{s}^{(\mathrm{st})}=\pi / 2-\varphi_{s}^{(\mathrm{st})}$, can be found as the stable (attracting) stationary points of the dynamical system (4a) and (4b). In this section, we shall generalize the results of [14] where the equilibria have been studied in relation to the anchoring ratio $w=w_{e} / w_{s}=W_{e} / W_{s}$. In particular, our analysis enables us to relax the constraints requiring the anchoring parameter $w_{s}$ to be small $\left(\left|w_{s}\right|<0.5\right)$ and the phase shift to be zero, $\varphi_{m}=0$.

2.1.1. Branches of Stationary States. Equations for the stationary solutions of the system (4a) and (4b) can be written in the following form:

$$
\begin{gathered}
\sin 2\left(\varphi_{e}+\psi_{s}\right)=\frac{\psi_{s}}{w_{s}}, \\
\cos 2\left(\varphi_{e}+\psi_{s}\right)=-\frac{\mu}{w_{s}} \sqrt{w_{s}^{2}-\psi_{s}^{2}}, \\
\sin 2\left(\varphi_{e}-\varphi_{m}\right)=\frac{\psi_{s}}{w_{e}}, \\
\cos 2\left(\varphi_{e}-\varphi_{m}\right)=\frac{\nu}{w_{e}} \sqrt{w_{e}^{2}-\psi_{s}^{2}},
\end{gathered}
$$


where $\mu$ and $\nu \in\{+1,-1\}$ are the indices numbering four different branches of the stationary states.

Similar to [14], our task is to examine the behavior of the stationary solutions when the anchoring ratio $w=$ $W_{e} / W_{s}$ varies whereas the anchoring parameter $w_{s}$ and the phase shift $\varphi_{m}$ are both kept constant. For this purpose, it is convenient to recast the stationarity equations (6) into the parametrized form

$$
\begin{aligned}
w & =W_{\nu}\left(t ; w_{e}\right) \\
& =t^{-1}\left\{t \cos 2\left(t+\varphi_{m}\right)+v \sqrt{w_{e}^{2}-t^{2}} \cos 2\left(t+\varphi_{m}\right)\right\},
\end{aligned}
$$

$$
\begin{aligned}
w^{-1} & =W_{\mu}^{(1)}\left(t ; w_{s}\right) \\
& =t^{-1}\left\{t \cos 2\left(t+\varphi_{m}\right)+\mu \sqrt{w_{s}^{2}-t^{2}} \cos 2\left(t+\varphi_{m}\right)\right\},
\end{aligned}
$$

where the surface director azimuthal angle $\psi_{s}$ plays the role of a parameter $-\left|w_{s}\right| \leq t \equiv \psi_{s} \leq\left|w_{s}\right|$. Then, the solutions of the equation

$$
\frac{1}{W_{\mu}^{(1)}\left(t ; w_{s}\right)}=W_{\nu}\left(t ; \frac{w_{s}}{W_{\mu}^{(1)}\left(t ; w_{s}\right)}\right) \Longrightarrow t \in D_{\nu \mu}
$$

form the branch of stationary points $D_{\nu \mu}$, and we can use (7b) to derive the relations

$$
\psi_{s}^{(\mathrm{st})}(w)=\left\{\begin{array}{l}
w=\frac{1}{W_{\mu}^{(1)}\left(t ; w_{s}\right)}, \\
\psi_{s}^{(\mathrm{st})}=t
\end{array} \quad t \in D_{\nu \mu}\right.
$$

representing the dependence of the stationary values of the surface director angle on the anchoring ratio in the form of the parametrized curve in the $w-\psi_{s}$ plane. Similarly, in the $w-\psi_{e}$ plane, the curve

$$
\begin{aligned}
& \psi_{e}^{(\mathrm{st})}(w) \\
& =\left\{\begin{array}{r}
w=\frac{1}{W_{\mu}^{(1)}\left(t ; w_{s}\right)}, \\
\psi_{e}^{(\mathrm{st})}=t+2^{-1}\left[\mu \times \arcsin \left(\frac{t}{w_{s}}\right)\right. \\
\left.+\frac{(1-\mu) \pi}{2}\right],
\end{array} \quad t \in D_{\nu \mu}\right.
\end{aligned}
$$

describes the corresponding branch of stationary easy axis angles.

We now pass on to the stability analysis of the stationary states. Following the standard approach $[16,17]$, the criteria of linearized stability are formulated in terms of the linearization matrix of the system (4a) and (4b), in the neighborhood of the fixed point $\left(\varphi_{e}^{(\mathrm{st})}, \psi_{s}^{(\mathrm{st})}\right)$,

$$
\mathbf{H}=\left(\begin{array}{ll}
1 & 0 \\
0 & \gamma
\end{array}\right) \cdot \boldsymbol{\Lambda}, \quad \boldsymbol{\Lambda}=-\left.\left(\begin{array}{ll}
\frac{\partial \Phi}{\partial \varphi_{e}} & \frac{\partial \Phi}{\partial \psi_{s}} \\
\frac{\partial \Psi}{\partial \varphi_{e}} & \frac{\partial \Psi}{\partial \psi_{s}}
\end{array}\right)\right|_{\varphi_{e}=\varphi_{e}^{(\mathrm{st})}, \psi_{s}=\psi_{s}^{(\mathrm{st})}}
$$

so that, in the case of two-dimensional systems, the stability conditions are given by

$$
\operatorname{Tr} \mathbf{H}>0, \quad \operatorname{det} \mathbf{H}>0 .
$$

The expression for the matrix $\Lambda$ evaluated for the branch of stationary points $D_{\nu \mu}$,

$$
\begin{aligned}
\left.\Lambda\right|_{t \in D_{\nu \mu}} & \equiv \Lambda_{\nu \mu}(t) \\
& =\left(\begin{array}{cc}
2 \nu \sqrt{w_{e}^{2}-t^{2}} & -1 \\
2 \mu \sqrt{w_{s}^{2}-t^{2}} & 1+2 \mu \sqrt{w_{s}^{2}-t^{2}}
\end{array}\right),
\end{aligned}
$$

can be derived with the help of (6).

2.1.2. Bifurcations of Equilibria. The surface director equilibria are represented by the solutions of (8) that meet the stability conditions (12). These conditions combined with the formula (13) for the matrix $\Lambda$ which enters the linearization matrix (11) can be analyzed using elementary methods to yield the following results: (a) $D_{++}$is the branch of stable stationary points (equilibria); (b) the branch $D_{--}$is unstable; and (c) for the two remaining branches $D_{\nu \mu}$ with $\nu \mu=-1$, $D_{-+}$, and $D_{+-}$, the stability conditions (12) are satisfied if and only if the determinant of the matrix $\boldsymbol{\Lambda}_{\nu \mu}$ is positive, $\operatorname{det} \boldsymbol{\Lambda}_{\nu \mu}>$ 0 . Thus, the inequality

$$
v \sqrt{\left[\frac{w_{s}}{W_{\mu}^{(1)}\left(t ; w_{s}\right)}\right]^{2}-t^{2}}\left(1+2 \mu \sqrt{w_{s}^{2}-t^{2}}\right)+\mu \sqrt{w_{s}^{2}-t^{2}}>0
$$

is the only stability condition that defines equilibria belonging to the branches $D_{-+}$and $D_{+-}$.

The bifurcation curves shown in Figures 1 and 2 represent the surface director and easy axis equilibrium angles computed using the parameterizations given in (9) and (10), respectively. The numerical procedure involves two steps: (a) solving $(8)$ at $(\nu, \mu) \in\{(+,+),(+,-),(-,+)\}$ and (b) isolating the stable points of $D_{\nu \mu}$ with $\nu \mu=-1$ from the unstable ones based on the stability criterion (13).

Figure 1 demonstrates the effect of the anchoring parameter $w_{s}$ on the bifurcation curves that describe dynamical behavior of the model (4a) and (4b) with relation to the anchoring ratio $w=W_{e} / W_{s}$ when the phase shift is zero, $\varphi_{m}=$ 0 . The case of small anchoring parameter with $w_{s}<1 / 2$ was studied in our previous paper [14] and is illustrated in Figures $1(\mathrm{a})-1(\mathrm{c})$.

It is seen that there is only the stationary value of the surface director angle, $\varphi_{s}^{(\mathrm{st})}=\pi / 2\left(\psi_{s}^{(\mathrm{st})}=0\right)$, provided that 


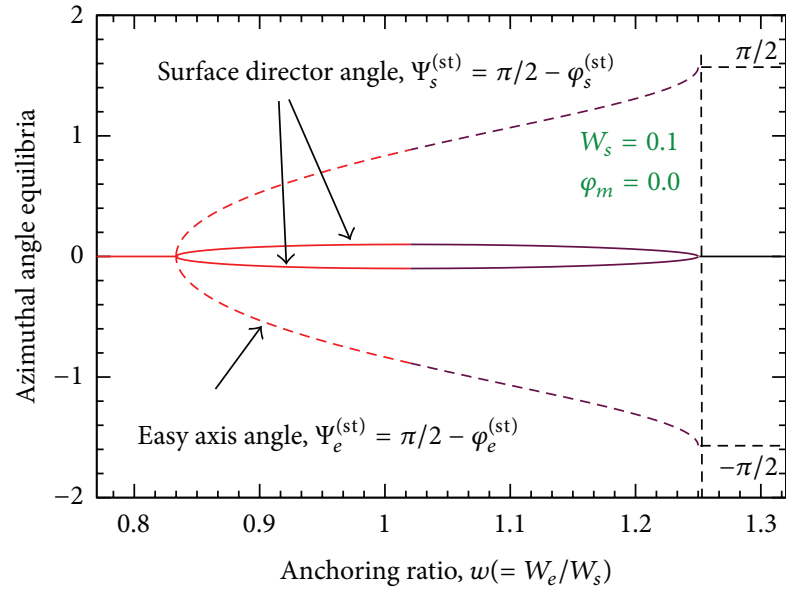

(a)

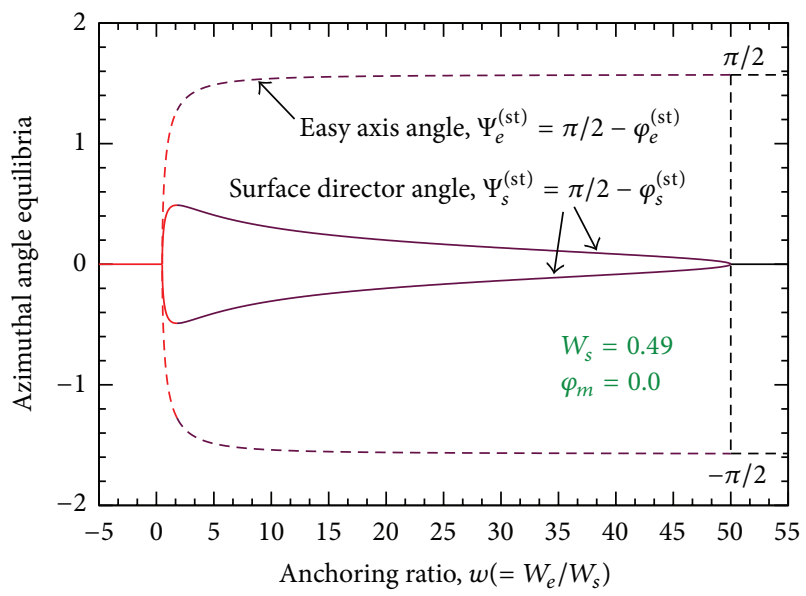

(c)

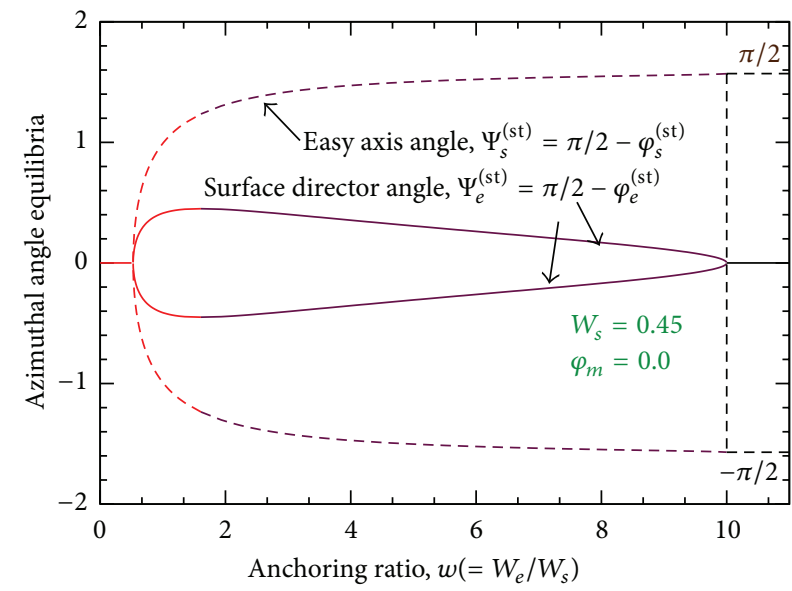

(b)

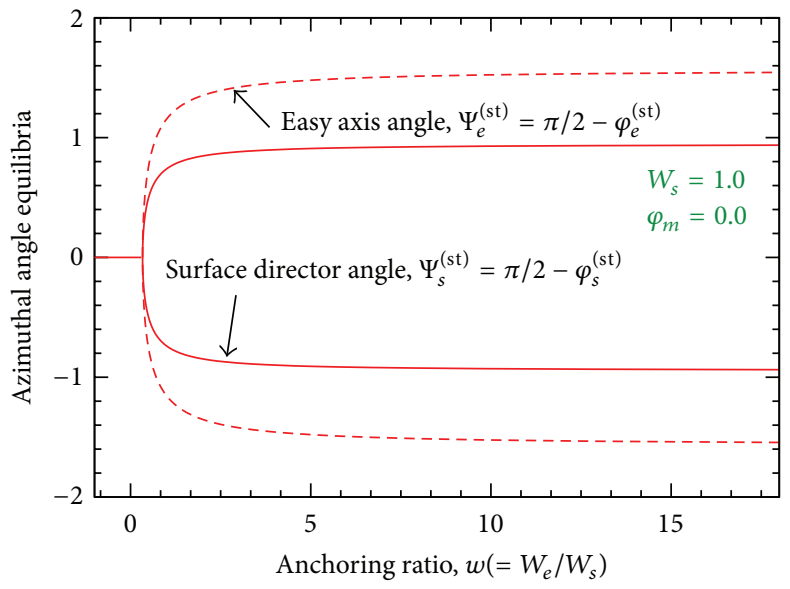

(d)

FIGURE 1: Bifurcation diagram for equilibria of easy axis and surface director azimuthal angles as a function of the anchoring ratio, $w=W_{e} / W_{s}$, at $\phi_{m}=0$. Four cases are shown: (a) $w_{s}=0.1$; (b) $w_{s}=0.45$; (c) $w_{s}=0.49$; and (d) $w_{s}=1.0$.

$w<w_{+}$or $w>w_{-}$, where $w_{+}=1 /\left(1 \pm 2 w_{s}\right)$ is the critical (bifurcation) value of the anchoring ratio. The equilibrium values of the easy axis angle in these two regions are $\varphi_{e}^{(\mathrm{st})}=$ $\pi / 2\left(\psi_{e}^{(\mathrm{st})}=0\right)$ at $w<w_{+}$and $\varphi_{e}^{(\mathrm{st})}=0\left(\psi_{e}^{(\mathrm{st})}=\pi / 2\right)$ at $w>$ $w_{-}$. The latter implies that, at large coupling parameter $W_{e}$ when $w>w_{-}$, the easy axis gliding is completely suppressed, whereas in the regime of weak coupling with $w<w_{+}$, the easy axis rotates approaching the stationary state of the surface director.

Referring to Figures $1(\mathrm{a})-1(\mathrm{c})$, when the anchoring ratio $w$ passes through the critical points $w_{ \pm}$, the stationary state $\varphi_{s}^{(\mathrm{st})}=\pi / 2$ becomes unstable and the pitchfork bifurcations [17] occur. So, for the surface director angle in the intermediate region with $w_{+}<w<w_{-}$, there are two symmetrically arranged stable stationary points. In this case, gliding is not suppressed, but by contrast to the regime of weak coupling, the equilibrium states of the easy axis and the surface director are no longer identical.

Now, we examine the important case of large anchoring parameter $w_{s}$ with $w_{s}>1 / 2$ that represents the regime of weak electric field (low voltage). At $w_{s}=1 / 2$, the largest critical value of the anchoring ratio $w_{-}$diverges, and it can be expected that bifurcation diagrams at $w_{s}>1 / 2$ are characterized by the only point of pitchfork bifurcation located at $w=w_{+}$. An example of such diagram is depicted in Figure 1(d).

In the zero-field limit with $E=0$, the electric coherence length $\xi$ and the parameter $w_{s}$ both become infinitely large, whereas the bifurcation point $w_{+}$decays to zero. From the formula (3), it is clear that the equilibrium angles at $w<w_{+}=$ $0\left(W_{e}<0\right)$ and $w>w_{+}=0\left(W_{e}>0\right)$ are $\varphi_{e}^{(\mathrm{st})}=\varphi_{s}^{(\mathrm{st})}=\pi / 2$ $\left(\psi_{e}^{(\mathrm{st})}=\psi_{s}^{(\mathrm{st})}=0\right)$ and $\varphi_{e}^{(\mathrm{st})}=\varphi_{s}^{(\mathrm{st})}=0\left(\psi_{e}^{(\mathrm{st})}=\psi_{s}^{(\mathrm{st})}=\pi / 2\right)$, respectively. So, reorientation of both the surface director and the easy axis is inhibited provided that the coupling constant $W_{e}$ is positive.

Application of the electric field facilitates the process of reorientation. At nonvanishing voltage, the threshold value of the anchoring ratio is positive, $w_{+}>0$, and increases with electric field. As is shown in Figure 1(d), in the region of strong easy axis coupling where $w>w_{+}$, the angles $\psi_{s}^{\text {(st) }}$ and $\psi_{e}^{(\mathrm{st})}$ differ in magnitude, $\left|\psi_{s}^{(\mathrm{st})}\right|<\left|\psi_{e}^{(\mathrm{st})}\right|$. So, even at large 


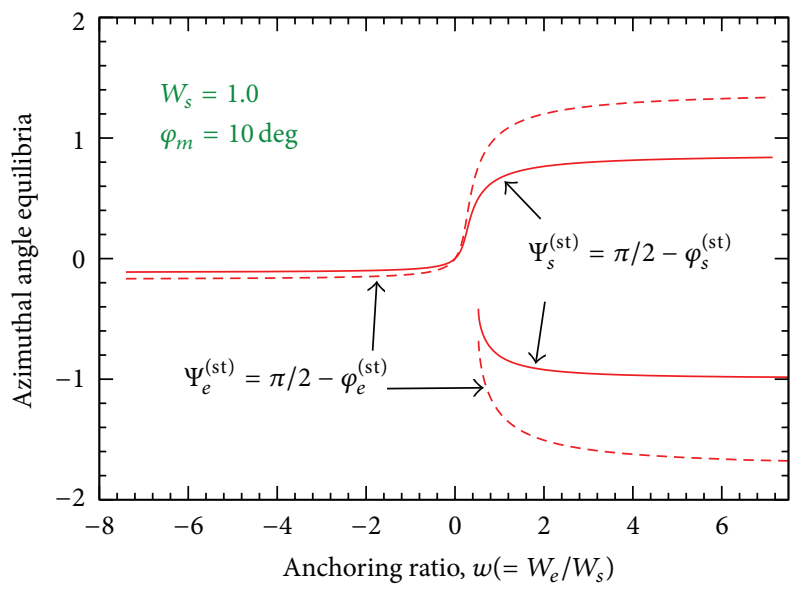

(a)

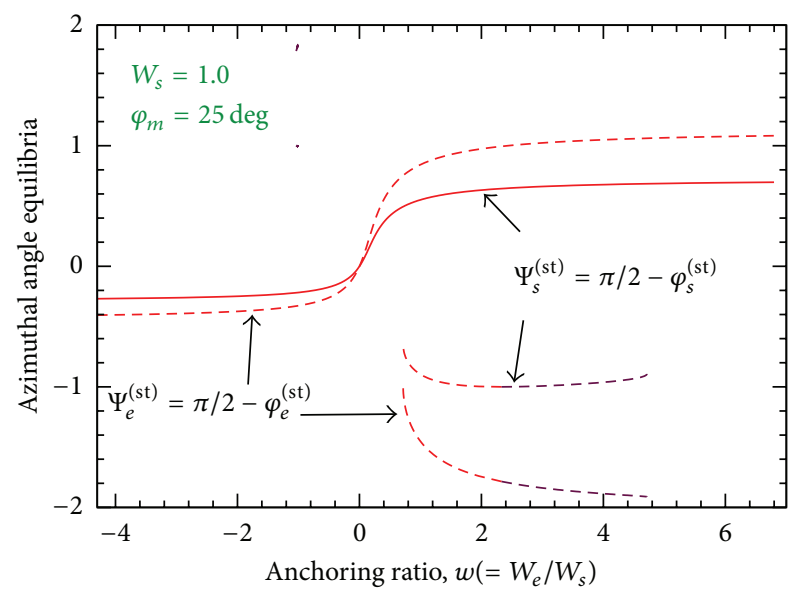

(c)

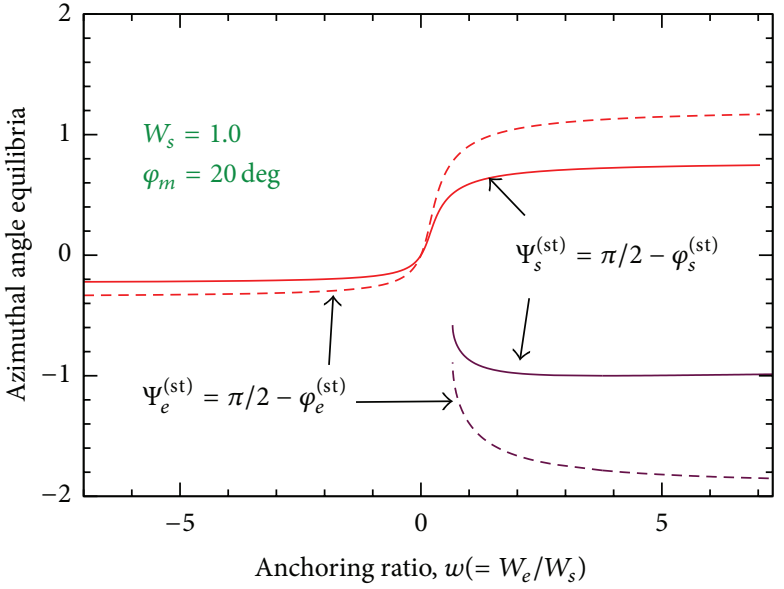

(b)

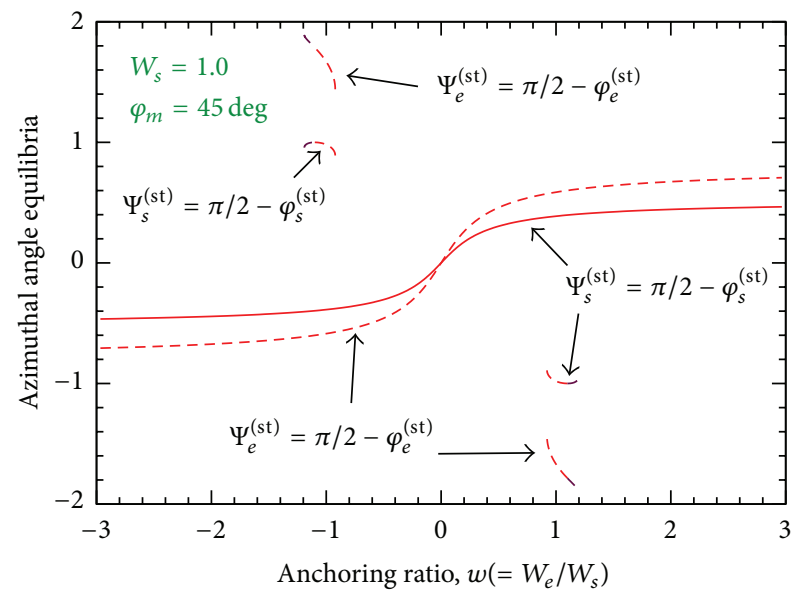

(d)

FIGURE 2: Bifurcation diagram for equilibria of easy axis and surface director azimuthal angles as a function of the anchoring ratio, $w=W_{e} / W_{s}$, at $w_{s}=1.0$. Four cases are shown: (a) $\varphi_{m}=\pi / 18$; (b) $\varphi_{m}=\pi / 9$; (c) $\varphi_{m}=5 \pi / 36$; and (d) $\varphi_{m}=\pi / 4$.

values of the anchoring ratio $w$, reorientation of the surface director takes place as its magnitude is below $\pi / 2$. The latter is not the case for the easy axis because the magnitude of the easy axis angle $\psi_{e}^{\text {(st) }}$ turned out to be very close to $\pi / 2$.

From the symmetry relation (5b), it follows that at $\varphi_{m}=$ 0 , bifurcation diagrams are invariant under reflection with respect to the $w$-axis: $\left(\psi_{s}^{(\mathrm{st})}, \psi_{e}^{(\mathrm{st})}\right) \rightarrow\left(-\psi_{s}^{(\mathrm{st})},-\psi_{e}^{(\mathrm{st})}\right)$ (see Figure 1). Generally, this is no longer the case for nonzero phase shift.

Another important consequence of the symmetry relations (5a) and (5b) is that under the action of inversion in the origin of coordinates $\left(w, \psi_{s}^{(\mathrm{st})}, \psi_{e}^{(\mathrm{st})}\right) \rightarrow\left(-w,-\psi_{s}^{(\mathrm{st})},-\psi_{e}^{(\mathrm{st})}\right)$ the diagram computed at $\varphi_{m}=\pi / 4-\Delta \varphi_{m}$ transforms into the one with $\varphi_{m}=\pi / 4+\Delta \varphi_{m}$. Hence we can restrict ourselves to a set of the diagrams with the phase shift ranged between 0 and $\pi / 4, \varphi_{m} \in[0, \pi / 4]$.

The bifurcation curves presented in Figure 2 illustrate the effect of the phase shift on the bifurcation diagrams at $w_{s}=1$. As evident from Figure 2(a), small variations of the phase shift play the role of a perturbation that splits the curves at the bifurcation point leading to the formation of the pattern consisting of two separated branches of equilibria. Such pattern is characteristic perturbed (imperfect) pitchfork bifurcations $[18,19]$.

From Figures 2(b)-2(d), it can be seen that when the phase shift increases, the low-lying branch of equilibria shrinks and, in the upper half of the diagram, additional branch develops. This branch grows with the phase shift up to the endpoint $\varphi_{m}=\pi / 4$ of the interval $[0, \pi / 4]$. As illustrated in Figure 2(d), at this point, the bifurcation diagram is center symmetric and the bifurcation curves remain intact under the action of inversion: $\left(w, \psi_{s}^{(\mathrm{st})}, \psi_{e}^{(\mathrm{st})}\right) \rightarrow\left(-w,-\psi_{s}^{(\mathrm{st})},-\psi_{e}^{(\mathrm{st})}\right)$.

\section{Model versus Experiment}

In the previous section, we have theoretically studied the general properties of the model. The results of our bifurcation analysis show that the photosteady states representing the easy axis and surface director azimuthal angles in the regime of photosaturation may undergo pitchfork bifurcations when the anchoring ratio $w=W_{e} / W_{s}$ varies. In this section, we 


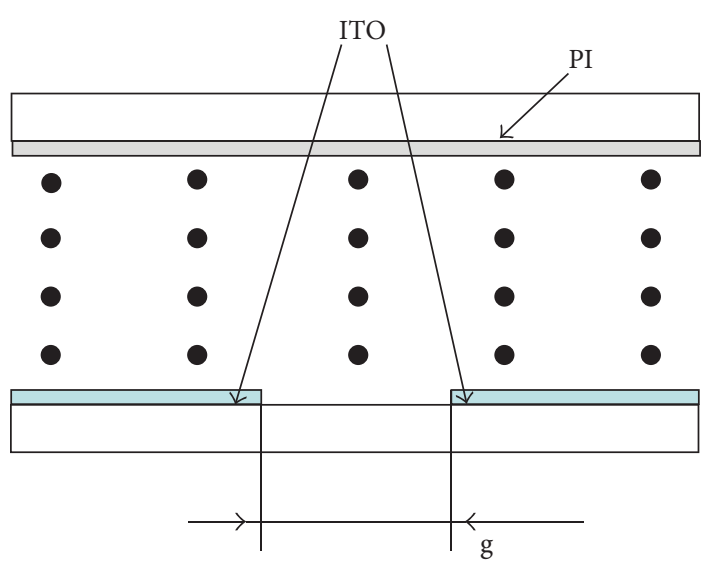

(a)

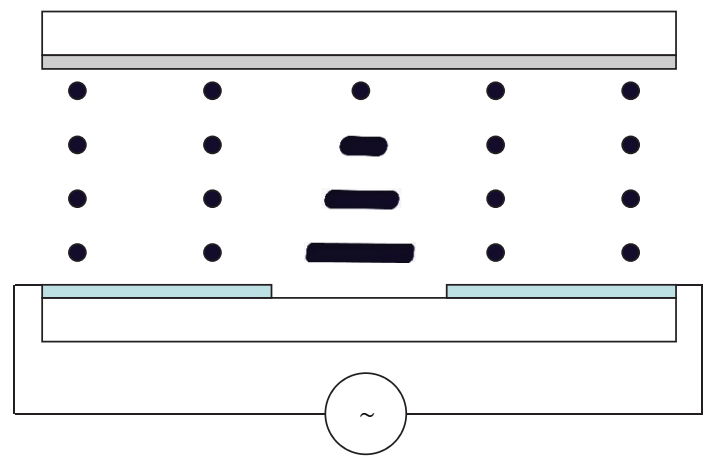

(b)

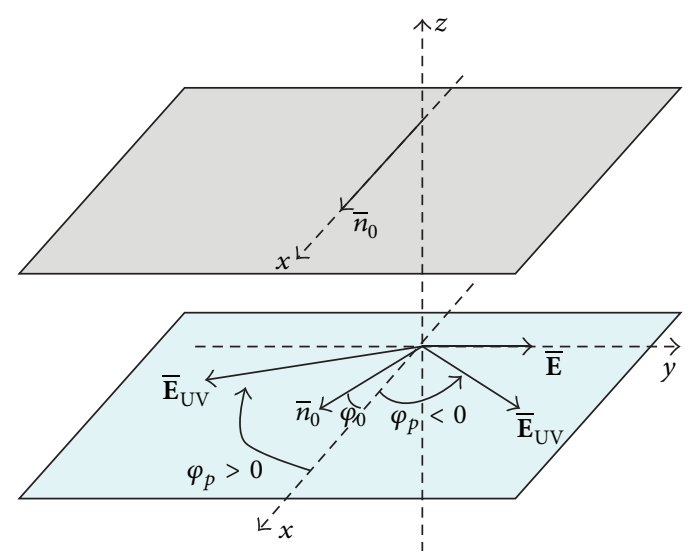

(c)

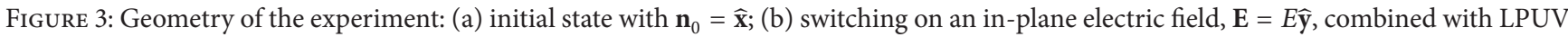
irradiation; (c) the system of reference: the polarization vector $\mathbf{E}_{\mathrm{UV}}$ of the LPUV light wave propagating along the normal to the substrate (the $z$-axis) is characterized by the polarization azimuth $\varphi_{p}$.

apply the model to interpret the experimental data, and the above results has been used as an effective tool to locate the intervals of physically meaningful model parameters. Though the part of the experimental results was recently reported in the letter [15], in order to make the paper self contained, they will be described at greater length and supplemented with new additional data.

3.1. Experimental Procedure. In our experiments, liquid crystal (LC) cells $(d=17.4 \pm 0.2 \mu \mathrm{m})$ of sandwich-like type were assembled between two amorphous glass plates. The upper glass plate was covered with a rubbed polyimide film to yield the strong planar anchoring conditions. In Figure 3, the direction of rubbing gives the easy axis parallel to the $x$-axis.

A film of the azobenzene sulfonic dye SD1 (Dainippon Ink and Chemicals) [7] was deposited onto the bottom substrate on which transparent indium tin oxide (ITO) electrodes were placed. The electrodes and the interelectrode stripes (the gap was about $g=50 \mu \mathrm{m}$ ) were arranged to be parallel to the $x$ axis (see Figure 3(a)).

As in [13-15], the azo-dye SD1 layer was initially illuminated by linearly polarized UV light (LPUV) at the wavelength $\lambda=365 \mathrm{~nm}$. The preliminary irradiation produced the zones of different energy dose exposure $D_{p}=0.07,0.14,0.27$, and $0.55 \mathrm{~J} / \mathrm{cm}^{2}$ characterized by relatively weak azimuthal anchoring strength. The light propagating along the normal to the substrates (the $z$-axis) was selected by an interference filter. Orientation of the polarization vector of UV light, $\mathbf{E}_{0}$, was chosen so as to align azo-dye molecules at a small angle of 4 degrees to the $x$-axis, $\varphi_{0} \approx 4$ deg (see Figure 3(c)).

The LC cell was filled with the nematic LC mixture E7 (Merck) in isotropic phase and then slowly cooled down to room temperature. Thus, we prepared the LC cell with a weakly twisted planar orientational structure where the director at the bottom surface $\mathbf{n}_{0}$ is clockwise rotated through the initial twist angle $\varphi_{0} \approx 4 \mathrm{deg}$ which is the angle between $\mathbf{n}_{0}$ and the director at the upper substrate (the $x$-axis).

As indicated in Figure 3(b), the director field deforms when the in-plane ac voltage $(U=100 \mathrm{~V}, f=3 \mathrm{kHz})$ is applied to the electrodes. In addition to the electric field, $E=$ $2 \mathrm{~V} / \mu \mathrm{m}$, the cell was irradiated with the reorienting LPUV light beam $\left(I_{\mathrm{UV}}=0.26 \mathrm{~mW} / \mathrm{cm}^{2}\right.$ and $\left.\lambda=365 \mathrm{~nm}\right)$ normally impinging onto the bottom substrate.

For this secondary LPUV irradiation, orientation of the polarization plane is determined by the polarization 
azimuth, $\varphi_{p}$, which is defined as the angle between the polarization vector of $\mathrm{UV}$ light, $\mathbf{E}_{\mathrm{UV}}$, and the $x$-axis. As indicated in Figure 3(c), we shall assume that positive (negative) values of the polarization azimuth, $\varphi_{p}>0\left(\varphi_{p}<0\right)$, correspond to clockwise (counterclockwise) rotation of the polarizer from the $x$-axis to $\mathbf{E}_{\mathrm{UV}}$.

Our experimental method has already been described in [13-15]. In this method, NLC orientational structures were observed via a polarized microscope connected with a digital camera and a fiber optics spectrometer. The rotating polarizer technique was used to measure the azimuthal angle $\varphi_{e}$ characterizing orientation of the easy axis. In order to register microscopic images and to measure the value of $\varphi_{e}$, the electric field and the reorienting light were switched off for about $1 \mathrm{~min}$. This time interval is short enough to ensure that orientation of the easy axis remains essentially intact in the course of measurements. The measurements were carried out at a temperature of $26^{\circ} \mathrm{C}$.

When the electric field $(E=2 \mathrm{~V} / \mu \mathrm{m})$ in combination with reorienting LPUV light of the intensity $I_{\mathrm{UV}}=$ $0.26 \mathrm{~mW} / \mathrm{cm}^{2}$ is applied for more than 120 minutes, we observed the memory effect. In this case, after switching off the field and light, the easy axis did not relax back to its initial state for at least few months.

The described technique allows to measure the values of $\varphi_{e}$ averaged over the illuminated area. In what follows, we also present some results concerning the angular distribution of local easy axis. For this purpose, we have used the image processing procedure where each irradiation step was followed by the analysis of the brightness of the pixels of the microscopic images in the regions within (outside) the interelectrode gaps. In our setup, the polarizing microscope was connected with 8-bit CCD camera of resolution $3504 \times 2336$ that made it possible to register the brightness for pixels of the size $0.2 \mu \mathrm{m} \times 0.2 \mu \mathrm{m}$.

The brightness was assumed to be proportional to the local intensity of light $I(x, y)$ transmitted through the cell placed between crossed polarizers. Since, after switching off electric field and LPUV light, propagation of the testing beam occurs in the Maugene regime, the expression linking the light intensity, $I(x, y)$, and the easy axis azimuthal angle, $\varphi_{e}(x, y)$, is $I(x, y)=I_{0} \sin ^{2} \varphi_{e}(x, y)$, where $I_{0}$ is the intensity of incident beam. This expression then can be used to obtain a map of $\varphi_{e}(x, y)$ of the azimuthal orientation of the angular field of the easy axis on the photoaligned layer. Note that a similar method was used in [20] to measure surface heterogeneities of the nematic director orientation.

3.2. Results. Figure 4 shows the microscopic images obtained at various times of irradiation by reorienting LPUV light for four different values of the polarization azimuth: $\varphi_{p}=$ $0,-24,-45$, and -65 degrees. In this case, the initial irradiation dose is fixed at $D_{p}=0.27 \mathrm{~J} / \mathrm{cm}^{2}$.

It can be seen that when the reorienting light is linearly polarized along the initial surface director $\mathbf{n}_{0}$ and $\varphi_{p}=$ 0 , the brightness of stripes within the interelectrode gaps is much higher as compared to the ones in the region outside the gaps where the electric field is negligibly small

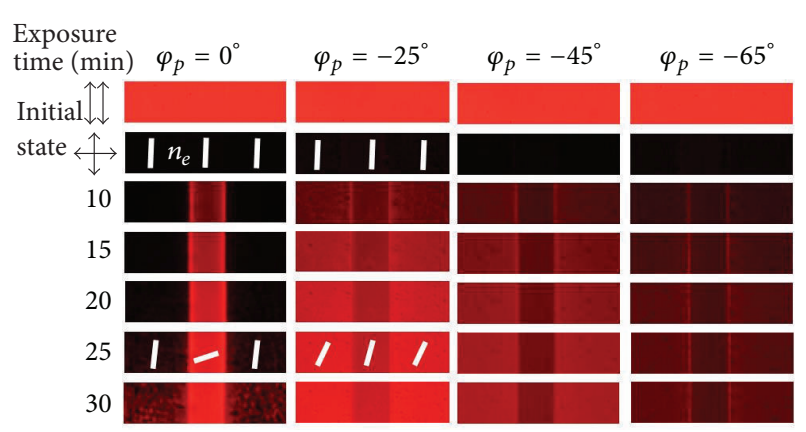

FIgURE 4: Microscopic images of the cell (filter with $\lambda=630 \mathrm{~nm}$ was used) in crossed polarizers for LPUV irradiation at different exposure times for various values of the polarization azimuth. The interelectrode gap $(g=50 \mu \mathrm{m})$ is indicated. The ac electric field is $E=2 \mathrm{~V} / \mu \mathrm{m}$, and the initial irradiation dose is $D_{p}=0.27 \mathrm{~J} / \mathrm{cm}^{2}$.

$E \approx 0 \mathrm{~V} / \mu \mathrm{m}$. So, in the case of vanishing polarization azimuth studied in $[13,14]$, we arrive at the conclusion that by contrast to the electrically assisted light-induced gliding, the purely photoinduced reorientation is almost entirely inhibited.

The latter is no longer the case for the reorienting light with nonzero polarization azimuth. Referring to Figure 4, at $\varphi_{p} \neq 0$, light-induced distortions of the surface director in the zero-field region located outside the gaps are very much more pronounced. It is also evident from the curves depicted in Figures 5(a), 6(a), and 7(a) representing the irradiation time dependencies of the easy axis angle measured at negative polarization azimuthal angles of the reorienting LPUV light.

In the zero-field curves, the easy axis angle increases with the irradiation time starting from the angle of initial twist, $\varphi_{0}$, and approaches the photo-steady state characterized by the photo-saturated value of the angle close to $\pi / 2+\varphi_{p}$. The curves describing the electrically assisted reorientation within the interelectrode gaps lie below the zero-field ones and reveal analogous behavior.

The data measured at the initial irradiation dose $D_{p}=$ $0.55 \mathrm{~J} / \mathrm{cm}^{2}$ for the polarization azimuths of the opposite sign, $\varphi_{p}>0$, (see Figures 5(b), 6(b), and 7(b)), show that in the zero-field region, the light-induced changes of the easy axis angle are negative and correspond to the counterclockwise rotation of the polarizer. As seen from Figures 5(b)-7(b), the dynamics of the easy axis in the presence of the electric field essentially differs from the one in the regime of purely photoinduced reorientation. In the interelectrode gaps, it turned out that the electric field prevails thus suppressing the tendency for the easy axis to be reoriented along the normal to the polarization vector of light $\mathbf{E}_{\mathrm{UV}}$.

Now, we discuss how the experimental data can be interpreted using the phenomenological model studied in Section 2. According to this model, the dynamics of the easy axis and surface director azimuthal angles, $\varphi_{e}$ and $\varphi_{s}$, is governed by the system of balance torque equations (1a) and (1b).

We can apply the formula (3) to fit the experimental data measured outside the interelectrode gaps. For $\varphi_{p}<0$ and $D_{p}=0.27 \mathrm{~J} / \mathrm{cm}^{2}$, the theoretical curves presented in Figures 5(a)-7(a) are computed at $t_{e} \approx 23 \mathrm{~min}$. The values of $\varphi_{m}$ are 


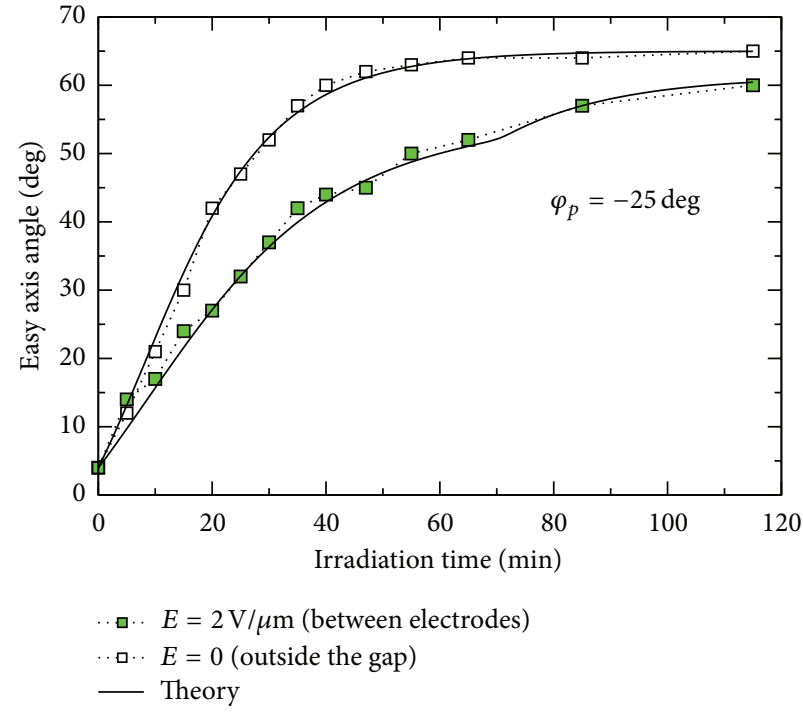

(a)

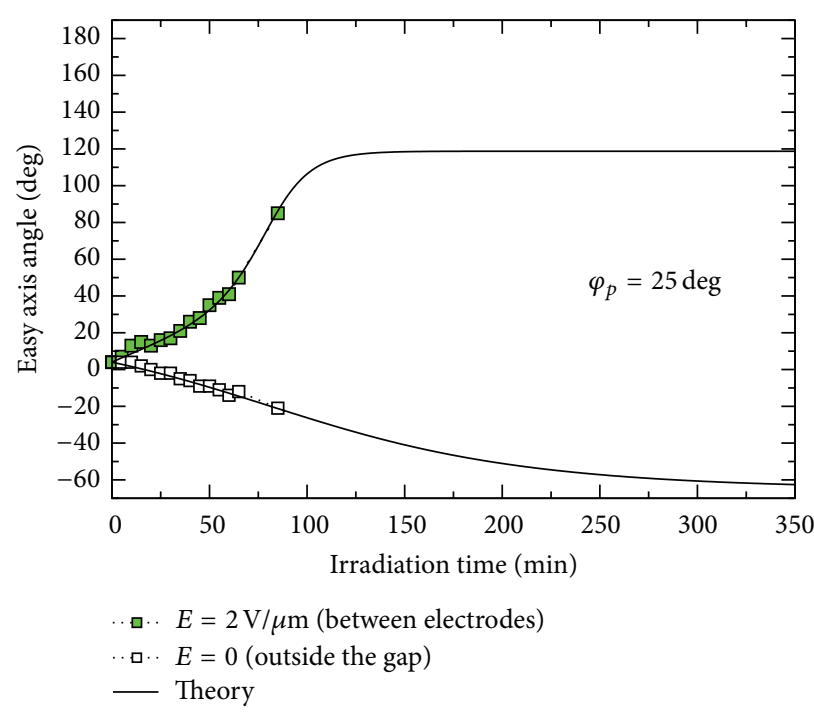

(b)

FIGURE 5: Easy axis angle as a function of the irradiation time measured for the reorienting LPUV light with different values of the polarization azimuth $\varphi_{p}$; (a) $\varphi_{p}=-25 \mathrm{deg}\left(D_{p}=0.27 \mathrm{~J} / \mathrm{cm}^{2}\right)$ and (b) $\varphi_{p}=25 \mathrm{deg}\left(D_{p}=0.55 \mathrm{~J} / \mathrm{cm}^{2}\right)$. Solid (open) squares represent the data measured in the regions within (outside) the interelectrode gaps where $E=2 \mathrm{~V} / \mu \mathrm{m}(E \approx 0 \mathrm{~V} / \mu \mathrm{m})$. The results computed from the phenomenological model by solving (1a) and (1b) are shown as solid lines.

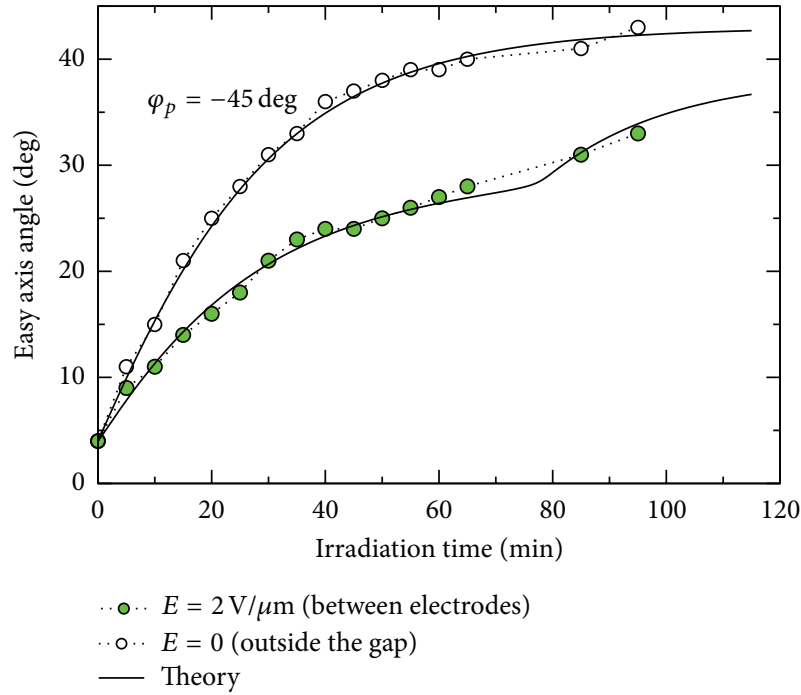

(a)

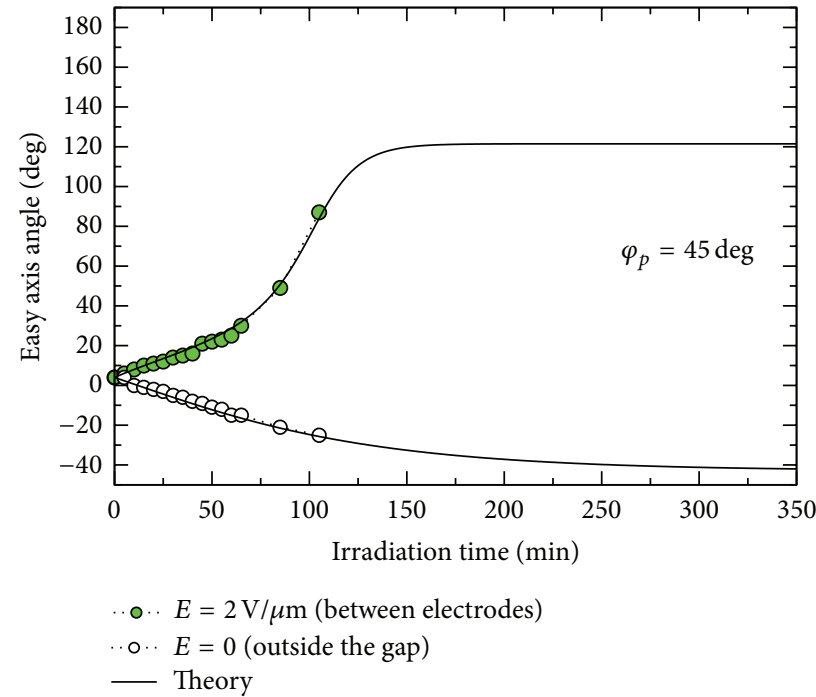

(b)

FIGURE 6: Easy axis angle as a function of the irradiation time measured for the reorienting LPUV light with different values of the polarization azimuth $\varphi_{p}$; (a) $\varphi_{p}=-45 \mathrm{deg}\left(D_{p}=0.27 \mathrm{~J} / \mathrm{cm}^{2}\right)$ and (b) $\varphi_{p}=45 \mathrm{deg}\left(D_{p}=0.55 \mathrm{~J} / \mathrm{cm}^{2}\right)$. Solid (open) circles represent the data measured in the regions within (outside) the interelectrode gaps where $E=2 \mathrm{~V} / \mu \mathrm{m}(E \approx 0 \mathrm{~V} / \mu \mathrm{m})$. The results computed from the phenomenological model by solving (1a) and (1b) are shown as solid lines.

$\varphi_{m}=25 \mathrm{deg}$ at $\varphi_{p}=-65 \mathrm{deg} ; \varphi_{m}=43 \mathrm{deg}$ at $\varphi_{p}=-45 \mathrm{deg} ;$ and $\varphi_{m}=65 \mathrm{deg}$ at $\varphi_{p}=-25 \mathrm{deg}$. Similarly, for the case where $\varphi_{p}>0$ and $D_{p}=0.55 \mathrm{~J} / \mathrm{cm}^{2}$, the results shown in Figures $5(\mathrm{~b})-7(\mathrm{~b})$ are computed at $t_{e} \approx 85 \mathrm{~min}$. In this case, the values of $\varphi_{m}$ are $\varphi_{m}=-38 \mathrm{deg}$ at $\varphi_{p}=55 \mathrm{deg} ; \varphi_{m}=-43 \mathrm{deg}$ at $\varphi_{p}=45 \mathrm{deg}$; and $\varphi_{m}=-65 \mathrm{deg}$ at $\varphi_{p}=25 \mathrm{deg}$.
In the presence of electric field (the region between the electrodes), the dynamical system (1a) and (1b) has to be solved numerically. As shown in the previous section, it is convenient to work with the system rewritten in the dimensionless form (4a) and (4b) which is characterized by the characteristic time $\tau_{e}=\gamma_{e} / K_{E}$, the viscosity ratio $\gamma=\gamma_{e} / \gamma_{s}$, 


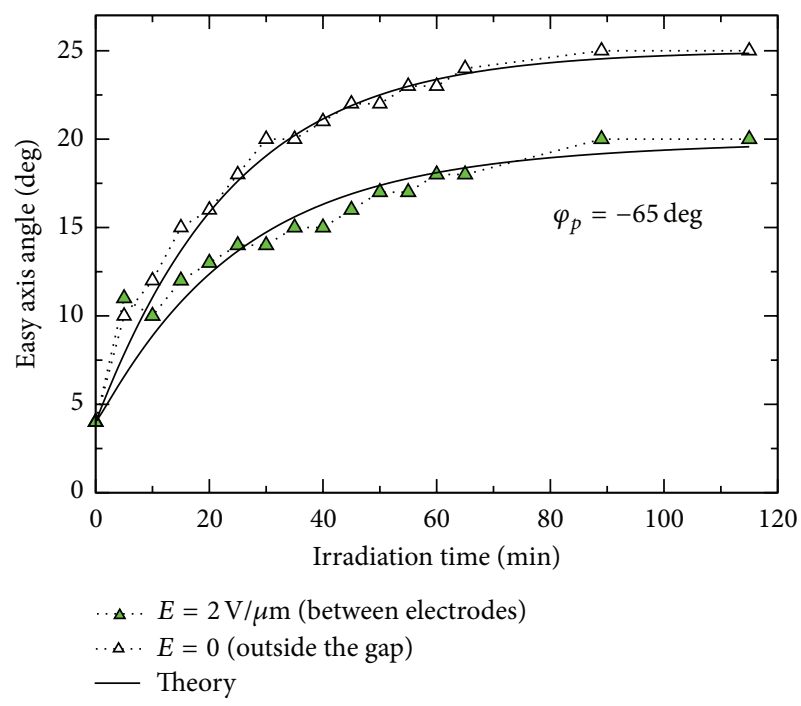

(a)

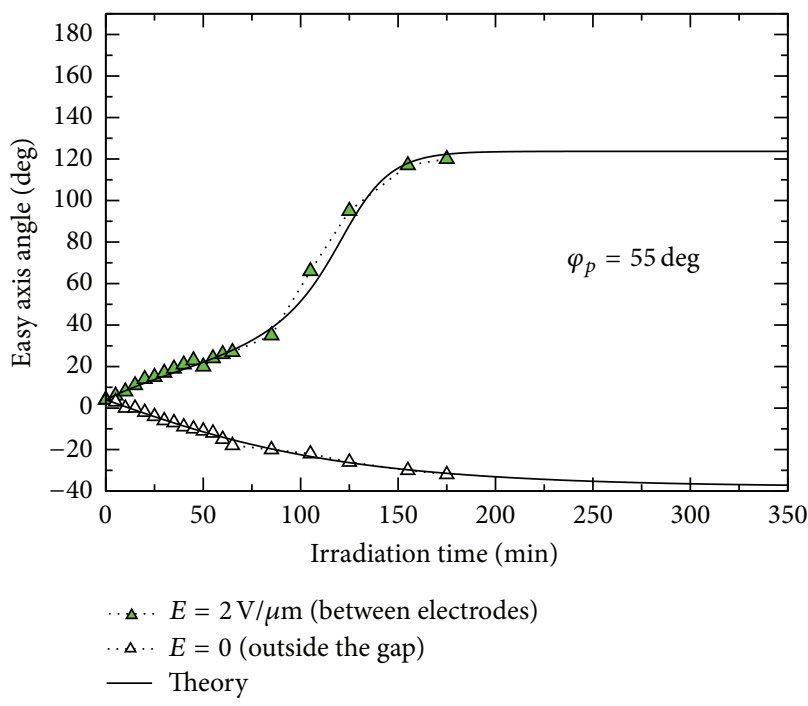

(b)

FIGURE 7: Easy axis angle as a function of the irradiation time measured for the reorienting LPUV light with different values of the polarization azimuth $\varphi_{p}$ (a) $\varphi_{p}=-65 \mathrm{deg}\left(D_{p}=0.27 \mathrm{~J} / \mathrm{cm}^{2}\right)$ and (b) $\varphi_{p}=55 \mathrm{deg}\left(D_{p}=0.55 \mathrm{~J} / \mathrm{cm}^{2}\right)$. Solid (open) triangles represent the data measured in the regions within (outside) the interelectrode gaps where $E=2 \mathrm{~V} / \mu \mathrm{m}(E \approx 0 \mathrm{~V} / \mu \mathrm{m})$. The results computed from the phenomenological model by solving (1a) and (1b) are shown as solid lines.

and the two dimensionless anchoring parameters $w_{e}=W_{e} /$ $\left(2 K_{E}\right)$ and $w_{s}=W_{s} /\left(2 K_{E}\right)$.

The parameters used for computing the curves are shown in Figures 5(a)-7(a) $\left(\varphi_{p}<0\right.$ and $\left.D_{p}=0.27 \mathrm{~J} / \mathrm{cm}^{2}\right): \tau_{e}=$ $230 \mathrm{~min}, \gamma=100$ (the gliding viscosity is typically several orders higher than the surface viscosity), $w_{e}=5$, and $w_{s}=1$. The values of $\varphi_{m}$ are $\varphi_{m}=25.1 \mathrm{deg}$ at $\varphi_{p}=-65 \mathrm{deg} ; \varphi_{m}=$ $35 \mathrm{deg}$ at $\varphi_{p}=-45 \mathrm{deg}$; and $\varphi_{m}=60 \mathrm{deg}$ at $\varphi_{p}=-25 \mathrm{deg}$. In Figures 5(b) $-7(\mathrm{~b})$ representing the case where $\varphi_{p}>0$ and $D_{p}=0.55 \mathrm{~J} / \mathrm{cm}^{2}$, the theoretical results are calculated at $\tau_{e} \approx$ $39.2 \mathrm{~min}, \gamma=100, w_{e}=1.1$, and $w_{s}=10$, and the values of $\varphi_{m}$, listed as follows: $\varphi_{m}=-41 \mathrm{deg}$ at $\varphi_{p}=55 \mathrm{deg} ; \varphi_{m}=$ $-45 \mathrm{deg}$ at $\varphi_{p}=45 \mathrm{deg}$; and $\varphi_{m}=-50 \mathrm{deg}$ at $\varphi_{p}=25 \mathrm{deg}$.

As it can be seen from Figures 5, 6, and 7, the computed curves are in good agreement with the experimental data. The results of fitting indicate that the initial irradiation dose has a profound effect on the anchoring parameters, whereas the electric field affects the polarization dependent phase shift $\varphi_{m}$.

More specifically, for the experimental data measured in the field-free region, the main result is that the characteristic time of purely photoinduced reorientation, $t_{e}$, strongly depends on the initial irradiation doses $t_{e} \approx 23 \mathrm{~min}$ at $D_{p}=$ $0.27 \mathrm{~J} / \mathrm{cm}^{2}$ and $t_{e} \approx 85 \mathrm{~min}$ at $D_{p}=0.55 \mathrm{~J} / \mathrm{cm}^{2}$. This result agrees with the prediction of the diffusion model [9] that the light-induced reorientation slows down with the initial ordering of azo-dye molecules.

Another effect of an increase in the initial ordering of the azo-dye film is that the anchoring energy strength $W_{s}$ is expected to grow with the initial irradiation dose $D_{p}$ [8]. This agrees with the results of fitting giving $w_{s} \approx 1$ at $D_{p}=$ $0.27 \mathrm{~J} / \mathrm{cm}^{2}$ and $w_{s} \approx 10$ at $D_{p}=0.55 \mathrm{~J} / \mathrm{cm}^{2}$.
The in-plane electric field generally counteracts the tendency for the easy axis to be oriented along the normal to $\mathbf{E}_{\mathrm{UV}}$. The rate of electrically assisted reorientation is found to be almost independent on both the sign of the polarization azimuth and the initial irradiation dose. The latter can be clearly seen from Figures 5(b)-7(b) where the curves representing the easy axis reorientation between the electrodes reach the regime of photosaturation much faster than the field-free ones measured outside the interelectrode gaps.

In closing this section, we consider another important effect of the initial ordering which is governed by the initial irradiation dose $D_{p}$ and takes place when the polarization azimuth equals zero, $\varphi_{p}=0 \mathrm{deg}$. As it was mentioned at the beginning of this section, this is the case where $\mathbf{n}_{0}$ is directed along $\mathbf{E}_{\mathrm{UV}}$ and unlike the above cases with nonzero azimuth, $\varphi_{p} \neq 0$ deg, the purely photoinduced reorientation of the easy axis was found to be completely suppressed.

The corresponding result of the theoretical analysis based on the diffusion model [9] is the threshold effect that occurs in azo-dye films with sufficiently high value of the initial ordering. For such films, the diffusion model predicts that the light-induced reorientation starts only if the intensity of the reorienting light exceeds its critical value (the threshold), $I_{\mathrm{UV}}>I_{c}$. Another theoretical prediction is that fluctuations of the easy axis orientation, $\mathbf{n}_{e}(0)$, have a destructive effect on the threshold behaviour.

Using the method described at the end of Section 3.1, we have performed a detailed analysis of local easy axis angular distribution in the regions within (outside) the interelectrode gaps at fixed polarization azimuth $\varphi_{p}=0 \mathrm{deg}$. The microscopic images of the cell at different exposure times, $t_{\text {exp }}$, and initial irradiation doses, $D_{p}$, are shown in Figure 8. 

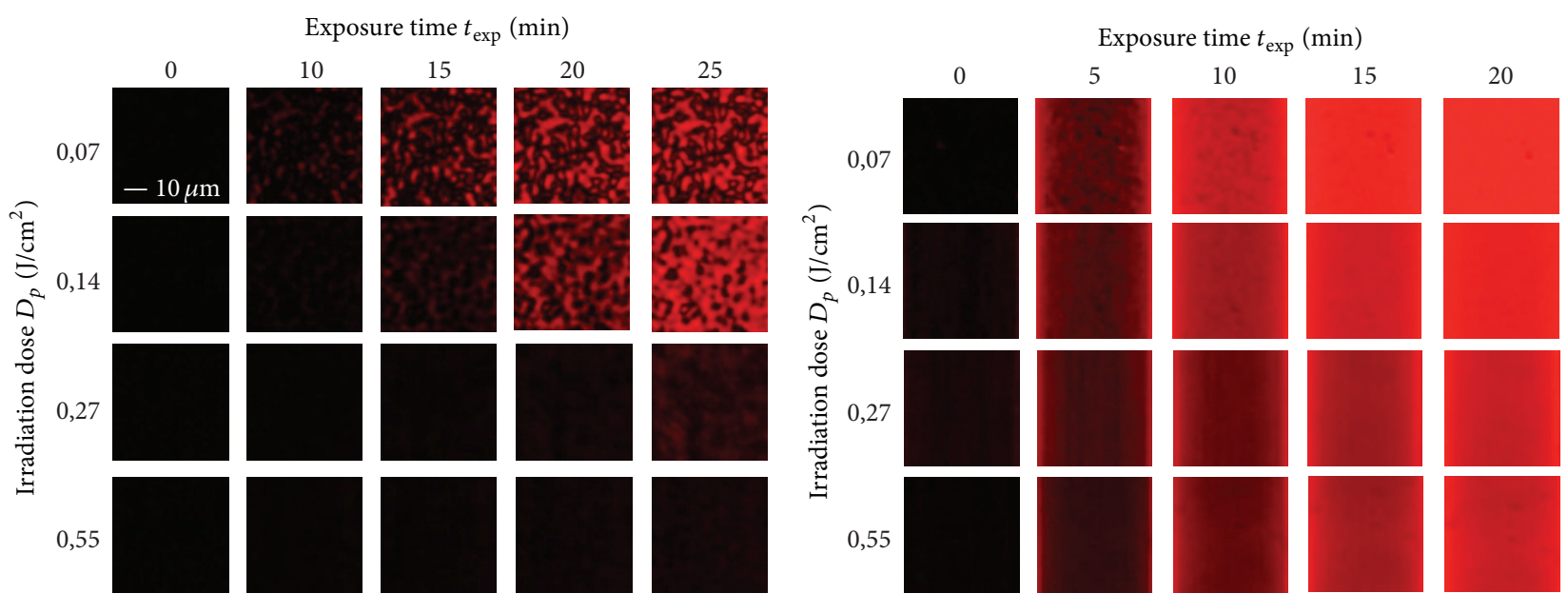

(a)

(b)

FIGURE 8: Microscopic images of the cells in crossed polarizers for LPUV irradiation at different exposure times with $\varphi_{p}=0$ deg and at various values of the initial irradiation dose $D_{p}$ used to prepare the cells. The images represent the regimes of (a) purely light-induced reorientation $(E=0 \mathrm{~V} / \mu \mathrm{m})$ and $(\mathrm{b})$ electrically assisted light-induced reorientation $(E=2 \mathrm{~V} / \mu \mathrm{m})$.

Figure 8(a) presents the microscopic images registered in the field-free regions where $E=0 \mathrm{~V} / \mu \mathrm{m}$. From Figure 8(a), it can be seen that the planar orientational structure in the cell is initially homogeneous, and noticeable light-induced reorientation takes place only if the initial irradiation dose is sufficiently low.

At $D_{p}=0.07 \mathrm{~J} / \mathrm{cm}^{2}$ and $D_{p}=0.14 \mathrm{~J} / \mathrm{cm}^{2}$, irradiation of the cell with reorienting LPUV light gives rise to the specklelike grainy pattern of bright and dark spots representing the heterogeneous distribution of light intensity. Such pattern is indicative of strong random variations of the twist angle (the angular domains) that can be described as disordering of the easy axis at the photoaligned substrate. In order to characterize the degree of disorder, for each pixel located at $(x, y)$ with the brightness proportional to $I(x, y)$, we have computed the value of the easy axis angle $\varphi_{e}(x, y)$ and summarized the results in the form of the histograms depicted in Figure 9.

Figure 9(a) shows how the histogram of the easy axis angle evolves with the exposure time in the field-free region $(E=0 \mathrm{~V} / \mu \mathrm{m})$ at $D_{p}=0.07 \mathrm{~J} / \mathrm{cm}^{2}$. It can be seen that, in this case, the degree of orientational easy axis disorder (the easy axis dispersion) grows as the exposure time increases. Referring to Figure 9(b), this is no longer the case in the presence of in-plane electric field.

The results for the regions within the interelectrode gaps where the light-induced reorientation is assisted by the electric field, $E=2 \mathrm{~V} / \mu \mathrm{m}$, are presented in Figures 8(b) and $9(\mathrm{~b})$. From these figures, it can be inferred that the in-plane electric field facilitates and speeds up the light-induced reorientation of the easy axis. In addition, as opposed to the case of field-free region, no disordering effects are observed within the interelectrode gaps (see Figure 9(b)).

\section{Discussion and Conclusions}

In conclusion, we have studied both theoretically and experimentally the effects of polarization azimuth in the electrically assisted light-induced azimuthal gliding of the NLC easy axis on the photoaligning azo-dye film. It is found that at nonzero polarization angle $\varphi_{p}$, the purely photoinduced reorientation takes place outside the interelectrode gaps, whereas when the polarization vector $\mathbf{E}_{\mathrm{UV}}$ is oriented along the initial surface director $\mathbf{n}_{0}$, such reorientation is almost entirely inhibited for highly ordered azo-dye films. For the field-free regime of reorientation with $\varphi_{p} \neq 0$, the easy axis reorients approaching the photosaturation limit close to the normal to the polarization vector. These results agree with the theoretical predictions of the diffusion model $[9,21]$ describing kinetics of photoinduced reordering in azo-dye films.

In the regions between electrodes with nonvanishing inplane electric field, the dynamics of reorientation slows down with the polarization azimuth and, as opposed to the case of purely photoinduced reorientation, the sense of easy axis rotation for the electrically assisted light-induced gliding is found to be independent of the sign of polarization azimuth. We have shown that the phenomenological torque balance model $[12,13]$ can be extended to interpret our data, whereas the theory developed in [9] cannot be directly applied to describe the effects of electric field.

We have theoretically studied the general properties of the model and performed a detailed bifurcation analysis of the dynamical system representing the model. It was found that the photo-steady states defined by the easy axis and surface director azimuthal angles in the regime of photosaturation may undergo pitchfork bifurcations when the anchoring ratio $w=W_{e} / W_{s}$ varies.

Our concluding remark concerns the results on the kinetics of purely photoinduced reorientation at $\varphi_{p}=0$ measured 


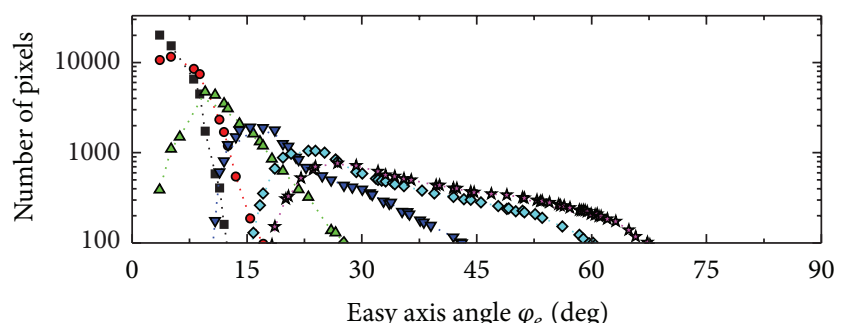

Exposure time ( $\mathrm{min})$

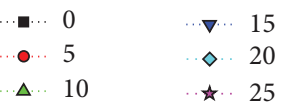

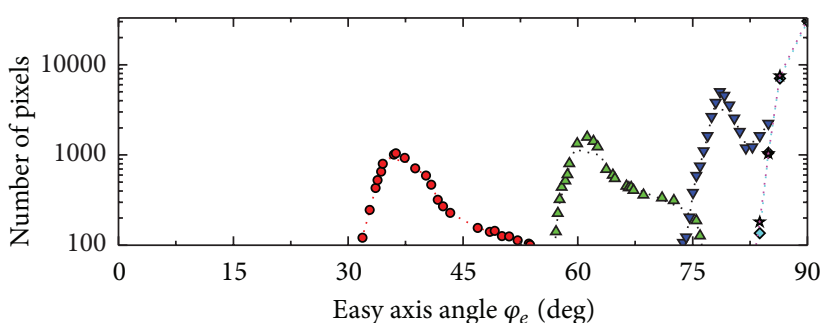

Exposure time (min)

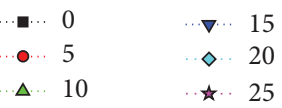

(b)

Figure 9: Histogram of the easy axis angle $\varphi_{e}$ at different exposure times $\left(\varphi_{p}=0\right.$ deg and $\left.D_{p}=0.07 \mathrm{~J} / \mathrm{cm}^{2}\right)$. (a) Purely light-induced reorientation $(E=0 \mathrm{~V} / \mu \mathrm{m})$; (b) electrically assisted light-induced reorientation $(E=2 \mathrm{~V} / \mu \mathrm{m})$.

as a function of the initial irradiation dose, $D_{p}$. There is a qualitative agreement between the experimental data and the theory [9] that predicts the threshold of reorientation, disordering effects, and sensitivity of the reordering kinetics to easy axis fluctuations. Clearly, these phenomena cannot be described by a model formulated in terms of averages.

On the other hand, theoretical considerations of [9] have to be generalized so as to incorporate the effects of electric field. A more systematic theoretical treatment is required to deal with the interplay of photoinduced ordering in azodye films, the anchoring energy effects, and the adsorptiondesorption processes underlying the gliding phenomenon. We hope that this work will stimulate further progress in the field.

\section{Acknowledgments}

This work was partially supported by grants from RF Ministry of Education and Science: 14.B37.21.0894, 14.B37.21.1198, and 14.B37.21.1914 and CERG Grants 612310 and 612409.

\section{References}

[1] T. J. Sluckin and A. Poniewierski, "Orientational wetting transitions and related phenomena in nematics," in Fluid Interfacial Phenomena, C. A. Croxton, Ed., Chapter 5, pp. 215-253, John Wiley \& Sons, Chichester, UK, 1986.

[2] B. Jerome, "Surface effects and anchoring in liquid crystals," Reports on Progress in Physics, vol. 54, no. 3, p. 391, 1991.

[3] G. Barbero and G. Durand, "Surface anchoring of nematic liquid crystals," in Liquid Crystals in Complex Geometries: Formed by Polymer and Porous Networks, G. P. Crawford and S. Zumer, Eds., Chapter 2, pp. 21-52, Taylor \& Francis, London, UK, 1996.

[4] W. M. Gibbons, P. J. Shannon, S.-T. Sun, and B. J. Swetlin, "Surface-mediated alignment of nematic liquid crystals with polarized laser light," Nature, vol. 351, pp. 49-50, 1991.

[5] D. Andrienko, A. Dyadyusha, Y. Kurioz, V. Reshetnyak, and Y. Reznikov, "Light-induced anchoring transitions and bistable nematic alignment on polysiloxane-based aligning surface," Molecular Crystals and Liquid Crystals Science and Technology Section A, vol. 321, no. 1, pp. 299-307, 1998.
[6] L. Komitov, K. Ichimura, and A. Strigazzi, "Light-induced anchoring transition in a $4,4^{\prime}$-disubstituted azobenzene nematic liquid crystal," Liquid Crystals, vol. 27, no. 1, pp. 51-55, 2000.

[7] V. G. Chigrinov, V. M. Kozenkov, and H.-S. Kwok, Photoalignment of Liquid Crystalline Materials: Physics and Applications, Series in Display Technology, John Wiley \& Sons, Chichester, UK, 2008.

[8] A. D. Kiselev, V. G. Chigrinov, and D. D. Huang, "Photoinduced ordering and anchoring properties of azo-dye films," Physical Review Letters E, vol. 72, no. 6, Article ID 061703, 12 pages, 2005.

[9] A. D. Kiselev, V. G. Chigrinov, S. V. Pasechnik, and A. V. Dubtsov, "Photoinduced reordering in thin azo-dye films and lightinduced reorientation dynamics of the nematic liquid-crystal easy axis," Physical Review Letters E, vol. 86, no. 1, Article ID 011706, 13 pages, 2012.

[10] A. Muravsky, A. Murauski, V. Chigrinov, and H.-S. Kwok, "Light printing of grayscale pixel images on optical rewritable electronic paper," Japanese Journal of Applied Physics, vol. 47, no. 8, pp. 6347-6353, 2008.

[11] V. P. Vorusev, H.-S. Kitzerow, and V. Chigrinov, "Azimuthal surface gliding of a nematic liquid crystal," Applied Physics Letters, vol. 70, no. 25, Article ID 3359, 3 pages, 1997.

[12] S. V. Pasechnik, V. G. Chigrinov, D. V. Shmeliova et al., "Slow relaxation processes in nematic liquid crystals at weak surface anchoring," Liquid Crystals, vol. 33, no. 2, pp. 175-185, 2006.

[13] S. V. Pasechnik, A. V. Dubtsov, D. V. Shmeliova, V. A. Tsvetkov, and V. G. Chigrinov, "Effect of combined action of electric field and light on gliding of the easy axis in nematic liquid crystals," Liquid Crystals, vol. 35, no. 5, pp. 569-579, 2008.

[14] A. V. Dubtsov, S. V. Pasechnik, A. D. Kiselev, D. V. Shmeliova, and V. G. Chigrinov, "Electrically assisted light-induced azimuthal gliding of the nematic liquid-crystal easy axis on photoaligned substrates," Physical Review E, vol. 82, no. 1, Article ID 011702, 6 pages, 2010.

[15] A. V. Dubtsov, D. V. Shmeliova, S. V. Pasechnik, A. D. Kiselev, and V. G. Chigrinov, "Effects of polarization azimuth in dynamics of electrically assisted light-induced gliding of nematic liquid-crystal easy axis," Applied Physics Letters, vol. 100, no. 14, Article ID 141608, 4 pages, 2012.

[16] Y. A. Kuznetsov, Elements of Applied Bifurcation Theory, vol. 112 of Applied Mathematical Sciences, Springer, New York, NY, USA, 2nd edition, 1998. 
[17] J. Guckenheimer and P. Holmes, Nonlinear Oscillations, Dynamical Systems, and Bifurcations of Vector Elds, vol. 42 of Applied Mathematical Sciences, Springer, New York, NY, USA, 1990.

[18] G. Iooss and D. D. Joseph, Elementary Stability and Bifurcation Theory, Undergraduate Texts in Mathematics, Springer, New York, NY, USA, 2nd edition, 1990.

[19] J. D. Crawford, "Introduction to bifurcation theory," Reviews of Modern Physics, vol. 63, no. 4, pp. 991-1037, 1991.

[20] M. Nespoulous, C. Blanc, and M. Nobili, "Orientational 8 disorder of a nematic liquid crystal," Physical Review Letters, vol. 104, no. 9, Article ID 097801, 2010.

[21] A. D. Kiselev, V. G. Chigrinov, and H.-S. Kwok, "Kinetics of photoinduced ordering in azo-dye films: two-state and diffusion models," Physical Review E, vol. 80, no. 1, Article ID 011706, 16 pages, 2009. 

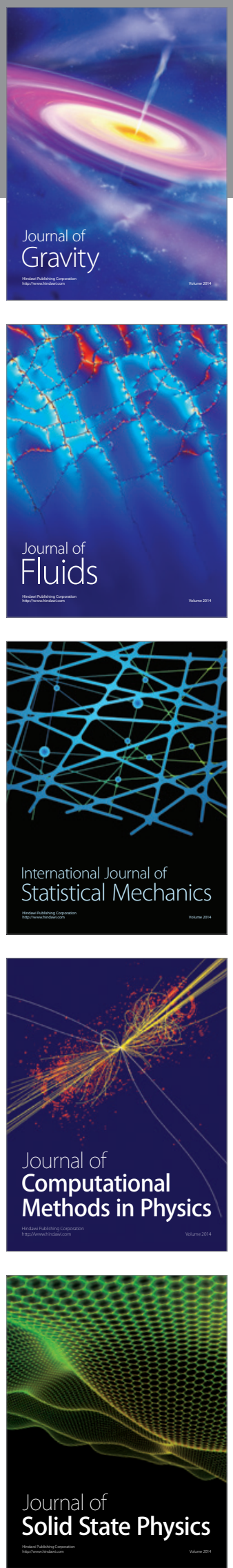

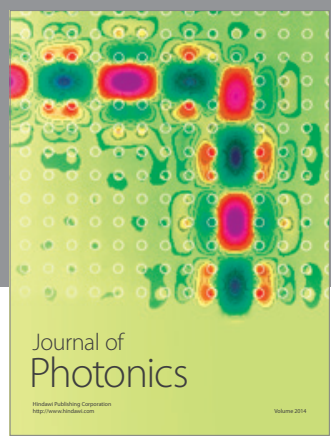

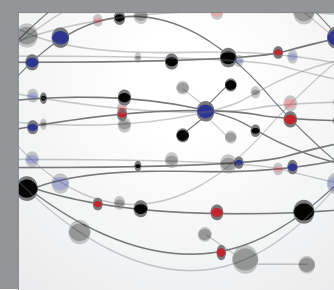

The Scientific World Journal

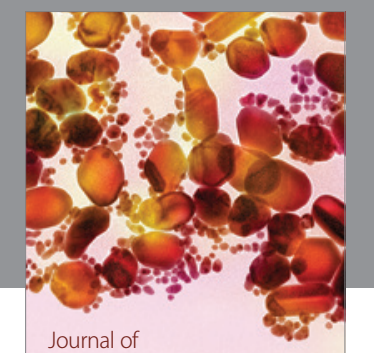

Soft Matter
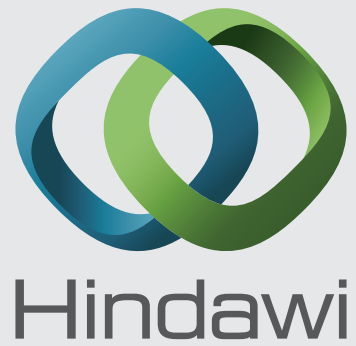

Submit your manuscripts at

http://www.hindawi.com
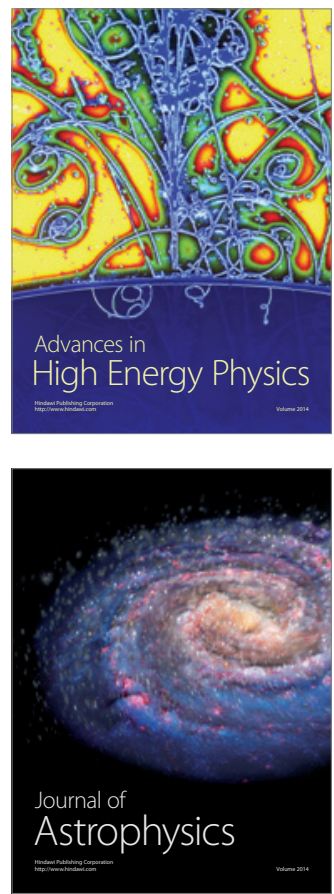
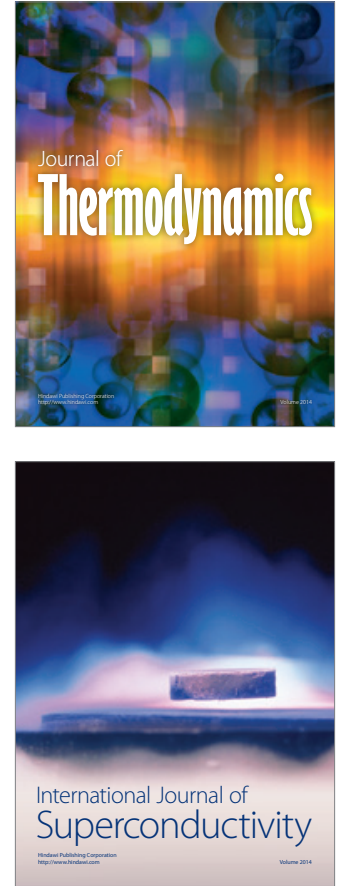
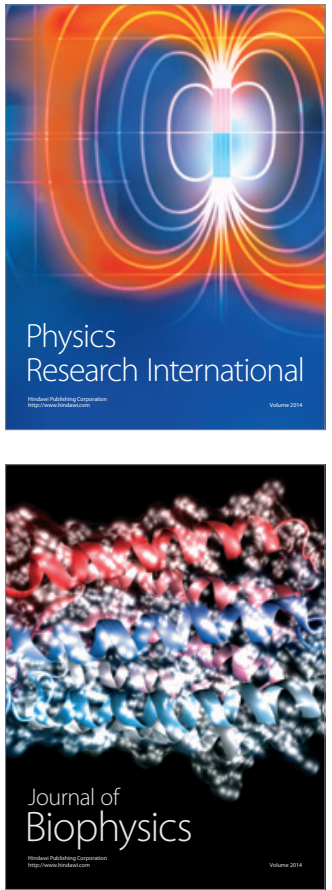
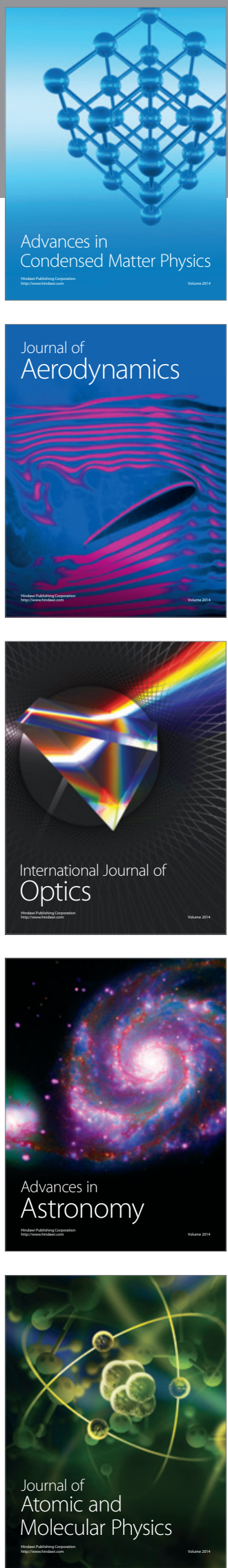